\title{
Francisolā
}

\section{INTEGRATION MORPHOSYNTAXIQUE ET SÉMANTIQUE DES ANGLICIMSES DANS LA PRESSE FRANÇAISE CONTEMPORAINE}

\author{
Mohamed Abdelbaki AHMED \\ Université du Sud de la Vallée, Egypte \\ mabaki_alsun@yahoo.fr \\ Article reçu le 15 octobre 2017 | révisé depuis le 18 octobre 2017 | accepté le 22 décembre 2017
}

\begin{abstract}
RÉSUMÉ. Cette étude s'inscrit dans le cadre de l'analyse linguistique synchronique des lexies anglaises présentes dans la presse française contemporaine. Cette étude examine les changements survenus dans la structure interne des mots sur le niveau syntaxique, morphologique et sémantique. Le corpus de cette étude comporte quelques journaux quotidiens de la presse française comme «Direct Matin», «Metro news » et « 20 Minutes». Cependant la consultation de quelques lignes d'autres journaux comme la «Libération», «Elle» et «Femme actuelle» est nécessaire pour mieux comprendre la nature des mots empruntés à l'anglais. Cette étude jette la lumière sur le rôle très primordial de la presse française dans la diffusion des anglicismes chez les locuteurs Français qui peuvent de leur côté aider à l'intégration morphosyntaxique et sémantique des lexies empruntées. Cette étude conclut que l'intégration des mots anglais devient suprême dans le discours quotidien des Locuteurs Français sur le niveau morphosyntaxique et sémantique. Bref, ce travail montre le rôle du contact langagier dans l'enrichissement lexical des langues en contact.
\end{abstract}

Mots- clés: Anglicisme, emprunt lexical, intégration, morphosyntaxe, sémantique.

\begin{abstract}
This study is part of the synchronic linguistic analysis of English words borrowed in the contemporary French press. It is an attempt to examine the changes in the internal structure of words on the syntactic, morphological and semantic level. A few daily newspapers of the French press like "Direct Matin", "Metro news" and "20 Minutes" have been selected as corpus of this study. Furthermore, a few lines from other newspapers like the "Liberation", "Elle", and "Femme actuelle", have been quoted occasionally to better understand the nature of the word borrowed from English. This study discusses the role of the French press in the spread of Anglicism among French speakers who can in turn help to make the morpho-syntactic and semantic integration of borrowed lexis. This study concludes that there is a high level of integration of English words in the daily discourse of French speakers in morpho-syntactic and semantic levels. In brief, this research reaches a conclusion of illustrating the role of linguistic contact in lexical enrichment of languages in contact.
\end{abstract}

Keywords : Anglicism, integration, lexical borrowing, morphosyntax, semantics. 


\section{INTRODUCTION}

La question de l'emprunt linguistique touche la plupart des langues vivantes du monde. Le français contemporain emprunte un nombre considérable de mots à la langue anglaise, celle-ci devient une langue universelle et toutes les autres langues du monde y puisent énormément des termes et des expressions. Nous allons mettre l'accent sur le processus du contact langagier entre le français et l'anglais et comment les mots anglais en français contemporain s'emploient beaucoup dans de nombreux domaines de la vie quotidienne. Nous montrons également comment ces mots migrateurs en français subissent quelques changements internes: syntaxiques, morphologiques et sémantiques. Bref cette étude s'inscrit dans le cadre de l'analyse linguistique synchronique des lexies anglaises empruntées à la presse française contemporaine et nous allons examiner les changements survenus dans la structure interne des mots sur le niveau syntaxique, morphologique et sémantique.

Pour bien comprendre le phénomène de l'intégration linguistique des mots anglais en français contemporain, il nous parait nécessaire de donner quelques remarques sur la notion de l'anglicisme et sa pénétration $\mathrm{du}$ corpus journalistique français.

L'anglicisme représente un emprunt dont la langue source est l'anglais soit d'Amérique ou d'Angleterre. Nous pouvons dire que tout élément provenant de la langue anglaise ou véhiculé par celle-ci est considéré comme anglicisme, quel que soit le degré de l'adaptation ou la date d'entrée dans la langue française (Engstrøm, 2005, p. 10).

Dans son étude sur la question de l'emprunt à l'anglais, Henriette Walter rappelle le rôle de l'anglais dans les domaines de la science, des techniques, de l'informatique, du cinéma, de la mode et du sport (Walter, 2001, P.21). Les anglicismes sont donc présents dans la langue française et ils deviennent des données importantes de l'histoire contemporaine de la langue et cet emploi de l'anglais par des francophones, dans des domaines tels que la science ou les affaires, progresse en France, en Belgique et en Suisse. (Rey, 2008, p.121).
D'autre part, le nombre et la fréquence des anglicismes varient selon les locuteurs et selon les domaines de spécialités. Certains domaines en dominent, comme l'économie mais aussi l'informatique et le sport. Les journalistes jouent un rôle primordial dans ce processus de l'adoption des lexies et des expressions anglaises dans le parler quotidien et professionnel des locuteurs francophones, puisque ces journalistes font introduire de nouveaux mots qui attirent le lecteur, ce qui garantit une rapide acclimatation dans la langue française, par le simple fait qu'il s'agit des lexies ressentis comme avantageux et utiles.

Concernant l'analyse de l'intégration interne des mots anglais dans la langue française, nous avons vérifié trois théories linguistiques différentes qui nous permettent de la compréhension du phénomène. La première théorie, celle de Haugen (1950) qui distingue deux grands instruments d'intégration, l'intégration sur le niveau phonologique et l'intégration morphologique. D'autre part, il parle d'importation phonologique, lorsque le son étranger est toujours respecté comme sa langue source alors qu'en cas de substitution phonologique, l'énonciateur effectue des efforts phonologiques en adaptant la prononciation du mot anglais au système phonologique français. De plus, Haugen mentionne une troisième catégorie intitulée cas intermédiaires. Il divise cette classe en calques et emprunts sémantiques qui portent des marques de substitution et d'importation. (Haugen, 1950, p.212)

La deuxième théorie qui nous intéresse est celle de Rey-Debove (1973) qui divise les éléments empruntés en deux catégories : les emprunts de nécessité et les emprunts de luxe. Les premiers s'attachent à des inventions ou à des techniques introuvables en France mais les derniers se rapportent à des objets ayant déjà un équivalent lexical français. Selon lui, il y a des phases pour l'intégration du terme étranger. Premièrement la phase métalinguistique dans laquelle le locuteur utilise le mot de sa langue maternelle et donne le synonyme dans l'autre langue. Dans la phase intermédiaire appelée la phase à connotation 
métalinguistique- autonymique, le locuteur prononce le mot étranger suivi d'un commentaire métalinguistique dans la langue maternelle et enfin la dernière phase intitulée la phase d'aboutissement qui marque l'intégration suprême dans la langue cible. (Rey-Debove, 1973, p. 110)

La troisième théorie sur l'adaptation de l'emprunt est celle d'Humbley (1974) qui distingue trois points pour l'étude des emprunts comme identification, modification, et causes. Il s'intéresse à la modification par rapport à la langue source. Pour l'identification, Humbley (1974, p.56) souligne que cela dépend du degré de la divergence ou de la convergence entre le terme emprunté et sa réception dans la langue cible. Selon lui, l'emprunt se manifeste à tous les niveaux de la langue: lexical, phonologique, graphique, morphologique et sémantique.

Ces trois théories nous retracent le chemin vers l'analyse interne des mots anglais dans la presse française contemporaine puisque le mot évolue dans le parler des locuteurs qui cherchent toujours à la modernité dans le choix des mots et le raffinement dans le style parlé.

Ce présent travail se base sur quelques études comme celles de Tournier (1998) qui classe les anglicismes par domaines et qui donne des explications sémantiques de ces lexies; de Walter (2001) qui explique l'histoire $\mathrm{du}$ contact franco-anglais et comment les langues peuvent échanger des mots et des expressions tout au long des siècles; de Engstrøm (2005) qui étudie les anglicismes de 1960 jusqu'en 2005 tout en étudiant l'adaptation morphosyntaxique des anglicismes et les motivations de leur intégration en français. Aussi, de Jedlickova (2009) qui parle de l'origine linguistique pour beaucoup de mots utilisés en français contemporain. Ce dernier travail clarifie les liens historiques unissant la France aux autres pays comme la Grèce, L'Espagne et l'Italie et comment les mots sont témoins oculaires du contact historique. Cette étude discute également la question de l'emprunt et si elle représente un enrichissement ou un appauvrissement de la langue d'accueil. la présente étude s'intéresse au processus de l'intégration linguistique des lexies anglaises dans le système linguistique de la langue française. Cette étude vise à mettre en relief l'adaptation des mots anglais par les locuteurs français qui se passionnent souvent par l'emploi de mots nouveaux. Le corpus choisi de cette étude semble être récent par rapport aux autres études antérieures qui ignorent totalement l'étude sémantique de nouveaux mots migrateurs en français. Dans cette recherche nous traitons comment les mots changent leur sens vers l'amincissement ou l'élargissement sémantiques.

Dans cette étude, nous essayerons de répondre à quelques questions qui semblent nécessaires: quels sont les éléments linguistique les plus employés chez le locuteur français? Ces anglicismes en français contemporain soumissent facilement au système morphosyntaxique français? Les mots empruntés possèdent -ils le même sens en anglais ou il accepte de nouvelles notions qui concernent la langue cible?

\section{MÉTHODE}

La réalisation de la présente étude s'appuie sur l'analyse linguistique des anglicismes dans la presse française contemporaine. Pour mieux décrire ce phénomène de l'emprunt à l'anglais, nous choisissions trois journaux quotidiens comme champ d'application: «Metro news », «Direct Matin », «20 Minutes ». Ces journaux sont gratuits et à la portée de tout le monde puisqu'ils sont distribués aux stations de métro, de RER et dans les gares de bus. Ces journaux datent de septembre 2014 jusqu'au février 2015. Ces journaux quotidiens comportent des domaines variés de la vie quotidienne: Sport, alimentation, mode, sciences techniques, cuisine, musique, etc. Bref nous avons collecté ces anglicismes dans les quotidiens français gratuits mentionnés ci-dessus à l'aide de quelques articles de journaux et magazines tels que : «Femme Actuelle» en 2014, «ELLE » en 2013, «Libération» en 2014 et c'est dans le but de compléter le corpus de l'étude et pour obtenir des exemples précis et suffisants pour l'analyse morphosyntaxique et sémantique 
des mots anglais intégrés en français contemporain.

Apres cette phase de recoupement des anglicismes dans la presse française, nous nous servons d'ouvrages de base de la question de l'emprunt linguistique. A cet égard, nous avons consulté les critères propres de l'analyse interne de l'emprunt chez Deroy (1956,p.67) Ces critères concernent les degrés de l'adaptation des noms, des adjectifs, des verbes, des pronoms et des prépositions. Deroy explique comment les nouveaux mots immigrés vers la langue cible subissent des changement phonologiques, morphologiques et sémantiques.

Nous nous limitons à l'analyse morphosyntaxique et sémantique des mots anglais dans la presse française contemporaine. Pour l'analyse morphosyntaxique, nous comparons entre les deux systèmes français et anglais concernant le traitement de genre, de pluriel, de suffixe, de préfixe et du système verbal. Pour l'analyse sémantique, nous montrons si les mots empruntés sont touchés d'un amincissement de sens, d'un élargissement de sens, ou d'un déplacement de sens tout en nous appuyant sur des exemples précis du corpus choisi.

\section{RÉSULTATS ET DISCUSSION Les éléments empruntés à l'anglais dans la presse française \\ Les noms}

le substantif est aisément emprunté que les autres éléments de la langue parce qu'il porte des notions et des connaissances de la langue source vers la langue cible et le locuteur de celle-ci a besoin d'exprimer ses nécessités linguistiques par des mots d'un sens complet ou à vrai dire c'est dans le lexique d'une langue que les emprunts sont les plus nombreux (Loubier, 2011, p.14.)

Le locuteur Français emprunte alors des noms anglais pour simplifier la description et pour éviter le malentendu qui peut être causé par l'emploi des mots longs et d'un sens un peu vague.

\section{Le nom en -ing}

Nous pouvons dire que les lexies ayant ce suffixe en anglais peuvent être des adjectifs, des participes, des gérondifs ou des noms, alors qu'en français ce suffixe s'attache principalement aux formes nominales. Voici des exemples :

[1] "Ces dernières années, le running a déferlé dans le quotidien des Français." (Japonnt, 2014)

[2] "Au programme des conseils pour commencer en douceur, un focus nutrition, cinq critères pour choisir ses baskets et notre shopping" (Japonnt, 2014).

[3] " Le spectateur ne nous avait pas vu venir: ni Philippe de Chauveron ni le casting n'étaient pas attendus comme le prochain Speilberg..." ("Medi Sadoun, sa vie après le bon Dieu", 2014).

Il nous parait à travers les trois exemples cités ci-dessus que le français adopte des mots anglais en suffixe ing sans aucun changement sémantique ou morphologique comme le mot shopping, parking, marketing et casting. Il y a aussi d'autres mots qui subissent de troncation comme running au lieu de running shoes et living au lieu de living room. Les locuteurs français peuvent à leur gré ajouter le suffixe ing à la fin du mot pour donner au mot un air anglais et pour l'ajout d'un nouveau sens lexical comme nous le verrons plus tard dans le mot footing.

\section{Les noms en -er, -or, -eur, -euse}

Nous pouvons souligner que le suffixe anglais er s'emploie pour désigner un être humain, une machine ou un animal. Ce suffixe n'apparaît pas dans les lexies d'une nature française mais seulement dans les emprunts à l'anglais comme dans les mots Shimmer, Slasher, sticker, poster, Dealer, Follower, Gamer (Picone, 1996, P. 334).

Nous comprenons que les lexies anglaises portant ce suffixe ne sont pas productives. Les locuteurs Français peuvent remplacer ce suffixe par celui qui se termine par -eur ou -euse comme équivalent possible à ce suffixe anglais comme nous le remarquons dans des mots comme rappeur, skateur, surfeur... 


\section{Les noms en -man}

Le suffixe man n'est pas d'une nature française mais d'une origine anglaise. Voici un exemple:

[4] "Mais il s'amuse tellement et c'est un showman." (Ouadhi, 2014)

Mais parfois le locuteur francophone peut ajouter ce suffixe pour d'autres lexies françaises afin de les donner une empreinte anglaise, ce qui représente un faux anglicisme comme dans le mot rugbyman. Ce mot est dérivé du mot rugby qui est un jeu qui oppose deux équipes de treize ou quinze joueurs consistant notamment à placer un ballon ovale derrière la ligne de but de l'adversaire. D'où le locuteur Français invente rugbyman qui est le joueur du rugby au lieu du mot anglais rugbyplayer, ce qui dénote que ce mot devient un faux emprunt.

\section{Des noms sans suffixe}

Nous avons relevé quelques noms sans suffixe dans la presse française contemporaine. Voici des exemples:

[5] "Alexandre de Lassus dévoile les meilleures adresses de la capitale pour lutter contre la tyrannie du stress" ("Paris en quête de Zenitude", 2014)

[6] "Ces adjectifs s'accordent très bien aux looks des rockers" ("Ils relookent leurs fans", ( 2014.

[7] "À toutes celles qui n'auraient pas trouvé leur robe, les 200 stands sont là..." ("le mariage est à l'honneur ce week-end", 2014).

Nous pouvons dire que l'ajout des déterminants du pluriel ou du singulier à ces mots anglais traduit une intégration suprême des anglicismes en français contemporain.

\section{Les adjectifs}

Nous pouvons dire que l'adjectif devient un élément très difficile à l'emprunt à cause de son manque d'autonomie et il se rapporte souvent à un nom. Selon Deroy l'emprunt de certains adjectifs en français contemporain prouve que le locuteur pénètre la mentalité étrangère notamment angloaméricaine. (Deroy, 1956 p.70 ). Voici des exemples:

[8] "Offre soumise à condition SFR ... disposant d'un niveau de service :
Gold, Silver ou platine. ( "On vous déroule le tapis rouge, " 2014)

[9] "Des canaux aux gratte-ciel et des vélos aux yellows cabs, un air hiphop siffle doucement". ("On vous déroule, le tapis rouge", 2014)

Nous pouvons comprendre que l'adjectif est un élément difficile à être emprunté à cause de ses problèmes de désinence et cela revient au fait exigeant l'accord entre l'adjectif et le nom auquel il se rapporte. Dans d'autres cas, nous rencontrons des adjectifs invariables :

[10] "C'est moins immersif, certes, mais aussi sans doute beaucoup plus fun à plusieurs" ("le salon devient un terrain de jeu", 2014).

Parfois, les adjectifs empruntés ont un suffixe anglais:

[11] "Le style Frenchy a connu une notoriété fulgurante essentiellement aux Etats-Unis"( "Stars grâce au web", 2014).

\section{Les verbes}

La presse française contemporaine emprunte un certain nombre des verbes anglais qui s'attachent essentiellement à des éléments techniques, sportifs ou cinématographiques, par exemple « scanner », « tester », « surfer », et scooter »:

[12] "Chaque bâtiment de cet espace comporte un code qui, une fois scanné, dévoile la structure finale sur son smartphone." (Quartier des Batignolles", 2014)

[13] "Ronaldo va tester notre défense, mais personnellement je m'en fiche un peu de tout ça." ("La nouvelle ère des Bleus", 2014)

[14] "Les bons sports, je les surfe. dit Paul Serin, Champion de France". ( "Les bons sports", 2014).

[15] " Ils s'étaient enfuis à scooter avant d'être cueillis un peu plus loin... ("Paris XIXe", 2014).

Il nous parait évidemment que les verbes anglais s'adaptent au système morphologique français à travers l'ajout des terminaisons verbales de la langue cible. Nous observons que certains verbes montrés ci-dessus étaient des noms en anglais mais le locuteur français les changent et les normalisent en verbes, ce qui indique la flexibilité et la richesse de la langue française 
à adopter et à recevoir favorablement les mots des autres langues.

\section{Les adverbes}

L'adverbe se définit comme un mot qui accompagne un verbe, un adjectif ou un autre adverbe pour en modifier ou en préciser le sens. (Dubois, 2002 p. 49). Les locuteurs qui empruntent des mots étrangers ne s'intéressent pas toujours à l'emprunt des adverbes ou des adjectifs parce que ce qui les passionne, c'est le mot, le terme ou l'expression qui satisfait leur sentiment intérieur de la distinction et de la promotion sociales. L'emprunt de l'adverbe exige une bonne connaissance de son sens et de sa place dans la langue source et son fonctionnement dans la langue cible. Nous n'avons pas trouvé d'exemples dans le corpus choisi sauf l'adverbe So:

[16] "으 chic, so basique: s'offrir un bon basique, c'est bien, savoir le porter, c'est encore mieux...." ("12 livres de mode...à la mode", 2013).

Ici la répétition de l'adverbe de la part du locuteur vise à attirer l'attention du destinataire et lui incite à suivre ses paroles par l'emploi des adverbes méconnues et peu utilisées des autres locuteurs pratiquant la même langue.

\section{Les sigles}

Nous pouvons dire que c'est à l'exemple de l'anglais que les sigles deviennent répandus en français et que plusieurs sigles sont empruntés directement ou indirectement à cette langue comme CD, DVD, SMS, MP3, USB. Le point de départ pour ce type d'emprunt a été le langage sportif puis le procédé s'est étendu aux autres domaines de la vie quotidienne. Dans le corpus choisi, nous avons observé l'emploi énorme de ces sigles. Voici des exemples:

[17] "La billetterie VIP des clients $\underline{\text { SFR }}$... ("On vous déroule le tapis rouge", 2014).

[18] "Ses données peuvent être transférés sur un ordinateur via un câble $\underline{\text { USB }}$ ". ("A l'heure des kids", 2014)

[19] "La comédie aux 12 millions d'entrées est sorti avant-hier en $\underline{D V D}$ agrémentée de scènes inédites et d'un bêtisier" ("Medi Sadoun, sa vie après le bon Dieur", 2014)
Nous constatons que les locuteurs français emploient des sigles qui appartiennent à la technologie et à la gestion. Ces sigles techniques sont répandus partout et à la portée aussi bien des spécialistes que des locuteurs simples.

\section{Les faux anglicismes}

Les pseudo- emprunts constituent une catégorie intermédiaire dans la classification car ils sont basés sur d'éléments d'une langue étrangère sans avoir un équivalent dans l'autre langue. Cependant il y a des lexies qualifiées comme pseudo emprunts puisqu'ils sont présents en anglais mais d'une nature sémantique presque différente en français. Nous rencontrons ces franglicismes dans le suffixe ing qui produit des lexies nominales en français, pour cela ce suffixe devient très productif en français et contribue à la fabrication de fausses lexies à titre anglais. Voici un exemple:

[20] "Et son propos de se faire encore plus édifiant, quand on sait que, lors d'un footing, la poitrine se déplace, verticalement, de 9 centimètres à chaque foulée." ("Le martien maximal" 2014)

Ce mot désigne promenade à pied faite dans un but hygiénique. Cette lexie n'existe pas en anglais en ce sens mais sa dérivation est logique à partir du verbe foot.

\section{Les hybrides}

Les hybrides sont des mots ou des expressions qui sont formés des éléments de la langue source en combinaison avec éléments de la langue cible. Nous pouvons rencontrer des mots anglais avec des éléments français dans des mots comme presse people, futur show, sweat- respirant, défi run, cybercafé... voici un exemple :

[21] " Pour courir à cette période de l'année, il faut se protéger des éléments.... Sweat respirant, coupevent.....Metronews a sélectionné la garde-robe...". ( "Prêts? Partez!", 2014)

Nous pouvons dire que l'hybridation devient un phénomène d'un usage superflu chez les jeunes hommes et par conséquent cet abus d'emploi le rend un peu refusé de la part de certains linguistes comme par exemples Berrendonner qui souligne que 
l'hybridation représente une structure syntaxique fautive, de type pléonastique qui comporte deux structures différentes de deux langues (Berrendonner, 1997, P.115). Aussi Forlot qui dénote que les mots hybrides ou emprunt mixte forment toutes sortes d'interférences fautives entre les structures anglaises et françaises (Forlot, 1999, p.113).

\section{Intégration morphosyntaxique des anglicismes \\ Les noms \\ Le genre}

Les substantifs empruntés à la langue anglaise n'ont pas de genre grammatical, mais en français, le locuteur doit donner au mot énoncé un genre d'une manière obligatoire. Par conséquent les énonciateurs français donnent toujours un genre au mot anglais emprunté. Le locuteur Français essaye d'ajouter un genre à la lexie anglaise selon le référent soit il est de sexe masculin ou féminin. Nous avons remarqué le grand emploi du genre masculin dans les mots anglais empruntés au français. Voici des exemples:

[22] "Le post qui aura le plus de signalements ne sera pas examiné en priorité." (" La censure selon Facebook", 2014)

[23] "L'éditeur Square Enix voit plus loin et a carrément annonce la création d'une filiale à New York consacrée $\underline{a u}$ cloud gaming." (Charciarek, N, 2014)

Mais ça n'empêche pas de trouver la présence du genre féminin. Voici des exemples:

[24] "Dans une interview fleuve accordée au Figaro, Nicolas Sarkozy expose ses idées." ( "Le candidat Sarkozy expose ses idées" 2014)

[25] "Partout où ne peut trouver un public enjoué au son du rap, de $\underline{l a}$ house et autres style". ("Un voyage culturel au cœur des cities" 2014)

Il nous semble que le genre féminin s'attache souvent à la nature du terme français équivalent c'est-à-dire le locuteur essaye de donner le genre féminin qu'il utilise pour le mot correspondant dans sa langue source. Humbley (1974, p.99) remarque que environ $90 \%$ des noms inanimés reçoivent le genre masculin. Il souligne qu'il y a des facteurs qui aident à adopter un genre féminin: (1) l'analogie avec le terme français correspondant; L'homographie partielle avec une lexie française ; et (3) la fin du mot qui éclaire la nature du genre.

\section{Le pluriel}

Le pluriel ne représente pas de difficulté pour les mots empruntés à l'anglais puisque la marque du pluriel $\mathrm{s}$ final est unique dans les deux langues et les mots empruntés peuvent avoir aisément cette marque. Voici des exemples:

[26] "Des looks Batman chez Colette" ("En bref", 2014)

[27] "Le groupe avait en effet annoncé qu'il rediffuserait des spots publicitaires..."("Pas de Pub après 20h" 2014)

Dans les mots composés empruntés à l'anglais, le locuteur met un s final à la fin du dernier mot. Voici un exemple :

[28] "Des extraits de spectacles, des one man shows, des concerts, des spectacles de rue...".(Les sorties culturelles à la fête" 2014).

Parfois nous rencontrons des noms toujours au pluriel comme kids

[29] "Tenue kids à la carte". ("Le bon plan du jour", 2014)

[30] "S'y massent des peoples, artistes, guest- stars, journalistes..." ("Un voyage culturel au cœur des cities", 2014) .

Il nous parait que le locuteur choisit le pluriel $\mathrm{du}$ mot selon son répertoire linguistique de sa langue source et ne s'intéresse pas au pluriel du mot dans la morphologie anglaise.

\section{Les adjectifs}

Nous observons que la majorité des adjectifs anglais en français restent toujours invariables et sont difficiles à devenir bien intégrés puisque l'adjectif se prête beaucoup moins à l'emprunt parce qu'il ne possède pas d'autonomie. Voici des exemples:

"Faire exploser le compteur à calories avec le traditionnel english breakfast." (Joly,2014) 
"Certes, mais aussi sans doute beaucoup plus fun à plusieurs". ("Le salon devient un terrain de jeu", 2014)

Nous remarquons que la présence du suffixe anglais ne permet pas à l'accord de ces adjectifs en français comme british, english, et aussi dans d'autres exemples, nous observons le transfert $\mathrm{du}$ nom en adjectif fun, ce qui indique que l'adjectif est un élément emprunté mais devient souvent invariable vu à sa nature anglaise rigide et qui ne se convient guère au système morphologique français. Bref l'influence d'une race sur une autre se manifeste surtout dans l'emprunt des adjectifs (Deroy,L, 1956, P.70.).

\section{Les verbes}

Nous pouvons dire que les verbes empruntés à l'anglais s'adaptent simplement au système morphologique français. Le locuteur ajoute aisément la désinence française à l'infinitif du verbe. Pour cela la plupart des verbes anglais prennent la flexion $\mathrm{du}$ verbe français du premier groupe et l'on peut obtenir des verbes conjugués aux différents temps verbaux du français. Voici des exemples au temps présent et passé composé:

[31]"le site ne scanne pas de mots- clés pour trouver des contenus..." ("Pédopornographie et terrorisme", 2014).

[32] "Les fashionistas ont flashé sur le poncho plaid de la maison..."(Demoulin, 2014).

[33] "Le quotidien américain a interviewé d'actuels et d'anciens cadres...". ("Le vrai récit du week-end noir", 2014)

\section{Le futur proche}

Le futur exprime toujours un événement à venir ou à vrai dire il indique une action qui se déroulera dans un avenir très proche du présent. Quand un verbe anglais se conjugue aux différents temps verbaux de la langue cible cela représente un bon indice de la part de la langue d'accueil vers adoption des verbes des autres langues:

[34] "Ronaldo va tester notre défense..."

("La nouvelle ère des Bleus", 2014)

Bref, nous comprenons que l'adaptation de différentes formes du verbe anglais au système morphologique français représente un degré suprême d'intégration des mots anglais dans le français contemporain. Cette adaptation se fait rapidement grâce à la désinence française qui facilite le processus de la flexion et de la conjugaison.

\section{Intégration graphique des anglicismes}

Nous pouvons remarquer que le concept d'orthographe nécessite la reconnaissance d'une norme écrite par rapport à laquelle on juge l'adéquation des formes que réalisent les sujets écrivant une langue ; l'orthographe suppose que l'on distingue des formes correctes et des formes incorrectes dans une langue écrite (Dubois, 2002, p.204). Il y a des critères pour juger l'intégration graphique des éléments empruntés comme la réécriture globale ou la francisation partielle des mots. Selon Humbley (1974, p.97), on observe très peu d'adaptation de l'orthographe des anglicismes aux règles françaises. D'ici nous comprenons que beaucoup d'anglicismes arrivent sans changement total de leur graphie dans la langue française et le locuteur français peut bien employer ces mots dans le parler quotidien comme nous l'observons dans de mots comme: SMS, Rugby, Rock, flash, film... Dans ce cas-là nous assistons à un xénisme lexical qui représente une unité lexicale constituée par un mot d'une langue étrangère et désignant une réalité propre à la culture des locuteurs de cette langue. (Quefflec, 2000, p.283).

Bref les mots anglais migrateurs vers le français respectent souvent la graphie française et subissent des formes de la francisation graphique partielle.

\section{Le trait d'union}

Les locuteurs Français ont tendance à ajouter des traits d'union aux mots composés empruntés à l'anglais comme nous l'observons dans de mots comme Bye-Bye, Ping-pong, Week-end, Dance-floor. Mais parfois le lecteur de la presse Française n'observe pas ce trait d'union dans quelques mots anglais comme Cloud gaming, Cheesecake. Nous pouvons dire que le trait d'union marque une bonne intégration des 
mots composés dans la graphie française, mais dans les cas de l'absence de ces traits, ça représente un choix de la part du journaliste vers l'adoption ou le refus de ces mots et les considérer comme des xénismes qui n'admettent aucune intégration graphique ou morphologique.

\section{L'Accent aigu}

L'accent est un signe graphique qui peut définir la voyelle et qui décrit son intonation. En français nous avons l'accent aigu et l'accent grave ou circonflexe. L'accent forme une spécificité graphique de la langue française et les mots empruntés à l'anglais gagnent ce changement graphique dans la forme des mots comme dans ces mots: musique électro, vidéomapping, Jeu vidéo.

Cette adoption de l'accent aigu pour les mots anglais dans l'écriture journalistique représente une forme d'intégration graphique des éléments empruntés et cela indique aussi la bonne volonté de la part des locuteurs vers la francisation des mots d'origine anglaise. En un mot, c'est cette adaptation qui assure la vitalité d'une langue devant les changements liés aux phénomènes d'hybridation et de métissage linguistique (Loubier, 2011, p.47).

\section{Intégration sémantique des anglicismes}

La sémantique est une discipline de la linguistique qui s'intéresse au sens d'un mot. Michel Bréal le décrit comme la relation de signification qui unit les mots aux choses, ou comme la relation existant entre les signes et leur utilisateurs (Vinzerich, 2007, p.88). Nous pouvons dire que les mots anglais qui immigrent vers la langue française changent parfois de sens. Ces mots gagnent un ou plusieurs de sens premiers et parfois ils sont nourris de nouvelles connotations sémantiques de la part de nouveaux locuteurs Français. Ce processus représente une forme d'évolution sémantique puisque tant qu'il y a des gens pour se servir d'une langue, elle est en perpétuel changement (Fagard, 2010, 147). Il y a des mots qui connaissent des périodes d'emploi plus ou moins généralisé. Parfois ils voient leur sens se diversifier ou se simplifier et il y a aussi certains mots qui sont oubliés et qui finissent par disparaître. C'est difficile de connaître quand un mot surgit ou quand il disparaît. Dans la suite, nous donnons des exemples où le mot anglais qui arrive en français gagne de nouvelles acceptions sémantiques: transfert $\mathrm{du}$ sens, élargissement de sens ou amincissement de sens. Voici des exemples des mots anglais qui admettent des changements sémantiques en français contemporain:

- Boy-friend

Le mot Boy-friend signifie un Homme ou un garçon avec qui l'on a une relation amoureuse, mais il s'agit d'une coupe particulière de pantalon, ample et un peu trop long (Tournier, 1998, p.159). Voici un exemple:

[35] "Le jean boyfriend est partout et du coup, ça vous titille forcément un peu de l'adopter ( Bonnet, 2017)

Dans cet exemple, nous observons que le mot anglais change son sens premier pour acquérir un nouveau sens particulier à la mode de la langue cible.

- Girl

En anglais, ce mot signale simplement une jeune fille, mais en français ce mot s'adresse à une jeune danseuse de musichall (Tournier, 1998, P.167). Voici un exemple:

[36] "Une fois le projecteur éteint! Mais, c'est pour mieux mettre en lumière les Girls Girls, Girls, venues enflammer le dance-floor pour le final." ("Un voyage culturel au cœur des cities" 2014).

Donc le mot perd le sens initial de la langue source pour acquérir un nouveau sens dans la langue cible.

- People

Ce mot signifie les gens en anglais mais en français ce mot est traité comme adjectif et un nom invariable. Ce mot, en français, porte le sens des ensembles des journaux et magazines qui consacrent leur première page et une bonne partie de leur contenu à des articles illustrés de photos concernant des gens célèbres de l'actualité comme vedettes de spectacle, du sport, de la politique, membres de la famille royale ou princière. Bref, Les peoples désignent les célébrités dont il est 
question dans les médias. Voici un exemple:

[37] "Ce beau soir de septembre, la file d'attente devant le théâtre... S'y massent des peoples, artistes, gueststars" ("Un voyage culturel au cœur des cities" 2014).

- $\underline{\text { Miss }}$

Ce mot souligne Mademoiselle, en parlant d'une Anglaise, d'une Américaine, mais dans le sens actuel du média français, ce mot dénote une jeune reine de beauté élue dans un concours comme Miss France, Miss Egypte (Tournier,1998, P.174):

[38] "Les miss Tour de France ont défilé en jaune, bardées de publicités." (Demoulin, 2014).

- City

En anglais, ce mot signale ville ou un centre historique de Londres où se trouve concentré l'essentiel de l'activité financière du Royaume-Uni, ou à vrai dire siège de nombreuses sociétés financières (Tournier, 1998, P.214). Dans la presse française contemporaine, l'emploi de ce mot signifie la capitale de Londres qui est considérée comme ville d'histoire et de culture:

[39] "C'est le monument le plus visité de la City, et son dôme de 108 mètres de haut est le deuxième plus grand d'Europe" (Genolini, 2014).

- Coach

Ce mot porte plusieurs sens. Il désigne un entraîneur sportif ou un répétiteur en argot des universités britanniques ou bien un wagon de chemin de fer (Tournier, 1998, p.27). Le locuteur Français utilise ce mot dans le domaine du sport à la place du mot entraîneur:

[40] "Le coach adjoint albanais a eu peur de mourir: l'entraîneur adjoint de l'Albanie, Altin Lala a eu très peur pour sa vie ..." (Baheux, 2014).

Donc un tel mot subit une restriction du sens de la part du locuteur qui le répète uniquement dans les milieux sportifs.

- Mobile

Ce mot signifie ensemble d'éléments construits en matériaux légers: tôle mince, plastique, fils métalliques et agencés de telle sorte qu'ils prennent des dispositions variées sous l'influence du vent ou de tout autre moteur ( Hachette, 1994, p.705). Ce mot a subi une restriction du sens puisque il désigne le téléphone portable dans le parler quotidien des Français. Voici un exemple:

[41] " Avec engagement de 12 mois en gardant votre mobile...." ("Un super forfait mobile à prix mini", 2014).

- $\underline{\text { Push }}$

En anglais, c'est un verbe qui signale pousser, mais en français ce mot a subi un changement total de sens puisqu'il signifie un message électronique envoyé à l'utilisateur d'un téléphone portable ou d'une tablette. Ce message s'attache à une application installée sur l'appareil de l'utilisateur. Nous pouvons lire une définition de Push de la part d'Isabelle Hanne dans son article intitulé Tentative de push:

[42] "Le push n'est pas une campagne de publicité pour faire venir des gens chez nous. Avec l'émergence du mobile, on est carrément dans le temps réel: nos lecteurs veulent être prévenus le plus vite possible". (Hanne, 2014).

Nous observons que le mot push transforme de sens et de nature puisqu'au lieu de pousser en anglais il signifie application technologique d'alerte et d'information et au lieu de sa nature verbale en anglais, il devient un nom masculin en français. Voici un autre exemple:

[43] "Pour l'instant, les éditeurs ne prennent pas beaucoup de liberté avec le push, notamment parce qu'il est envoyé à plusieurs centaines de milliers d'utilisateurs à chaque fois..." (Hanne, 2014).

- $\mathrm{Job}$

Ce mot est figuré comme travail ou profession en anglais mais en français il signale un travail rémunéré, qu'on ne considère généralement pas comme un véritable métier puisqu'il est de nature provisoire (Hachette, p.611). Voici un exemple

[44] "Vous cherchez un job, la ville de Paris recrute des accompagnements." ("La ville de Paris recrute des accompagnateurs", 2014) 
Donc ce mot gagne une spécialisation de sens d'une nature péjorative par rapport au sens employé dans la langue source.

Nous pouvons rencontrer beaucoup de problèmes sémantiques dans les faux anglicismes. Ces franglicismes sont construits d'un morphème ou d'un lemme dont l'origine anglaise donne faussement le lecteur ou le locuteur l'impression de l'étymologie anglaise de la totalité de la construction. D'autres mots que les locuteurs Français emploient dans le parler quotidien mais ils n'ont pas le même sens ou la même forme en anglais comme les mots: Zapping, smoking, relooking, brushing, parking, planning, camping, etc.

Il nous parait que les faux anglicismes causent beaucoup de problèmes sémantiques puisqu'ils ont une forme anglaise inventée par les locuteurs de la langue cible mais ils ne correspondent pas au même mot correct dans la langue source. Pour cela ces franglicismes ne peuvent pas être compris facilement pour les locuteurs Anglais car ces lexies représentent des sigles ou des mots décryptés et incapables d'être pratiqués hors le milieu linguistique français.

\section{CONCLUSION}

De ce qui précède, nous avons compris que la presse Française emploie intensivement des mots et des expressions empruntés à la langue anglaise, celle-ci domine un bon nombre de domaines notamment la technologie, le sport et la mode. Le locuteur Français peut emprunter beaucoup d'éléments linguistiques comme le nom, l'adjectif et le verbe. Ces anglicismes en français contemporain augmente d'une manière observée pour des raisons qui s'attachent à la volonté du locuteur ainsi qu'au journaliste.

Dans la partie qui concerne l'analyse morphosyntaxique, nous avons remarqué que les emprunts ont déjà gardé la forme de la langue source mais des ajustements orthographiques, comme l'accent aigu, le trait d'union, et grammaticales, comme l'ajout d'un genre et le remplacement de la désinence anglaise par la désinence française convenable, font normaliser les mots empruntés et les rendent plus admissibles et lisibles pour le locuteur Français. Pour le système verbal des emprunts à l'anglais, le locuteur Français ne trouve pas de problème pour l'adoption de ces verbes puisqu'ils sont conjugués dans la plupart du temps et sont traités comme des verbes du premier groupe.

D'ailleurs, nous avons remarqué que l'adjectif emprunté à l'anglais représente souvent un problème au locuteur débutant de la langue cible puisque le système morphologique anglais reste encore rigide par rapport aux adjectifs français comme nous l'observons dans des adjectifs comme Top, fun, english, et frenchy. Dans la partie qui concerne l'étude sémantique, nous avons découvert que les anglicismes employés dans les rubriques des journaux peuvent aussi soumettre au nouveau climat linguistique de la langue cible puisque des mots anglais empruntés peuvent accepter de nouveaux sens de la langue cible comme nous le voyons dans le mot: Boy-friend, Girl, Miss Job, People Push....

Bref, Ces franglicismes peuvent avoir un amincissement de sens ou un élargissement de sens. D'ici nous comprenons que les mots anglais empruntés au français évoluent de sens, ce qui dénote un degré suprême de l'intégration sémantique et morphosyntaxique. Bref les anglicismes sont témoins oculaires d'un contact langagier qui peut se manifester dans le parler quotidien du locuteur Français ainsi qu' aux articles journalistiques dont les thèmes sont souvent variés et récents.

\section{REMERCIEMENTS}

J'adresse mes remerciements très distingués au bureau des missions scientifiques en Egypte de m'avoir accordé une bourse de six mois à Paris. Cette bourse me permet d'un contact direct avec la langue et de fréquenter les Universités Françaises pour ma formation scientifique. Je remercie également mes professeurs égyptiens qui ne cessent de me tendre généreusement la main en cas de consultation scientifique.

\section{RÉFÉRENCES}

12 livres de mode...à la mode. (2013, 21 novembre). Elle, p.8. 
A l'heure des kids. (2014, 26 septembre). Direct Matin, p.26.

Baheux, R. (2014, 14 octobre). La coupe d'Europe est plus intense que le top 14. 20 Minutes, p.35.

Berrendonner, A (1997). Pléonasmes syntaxiques: dédoublement ou hybridation ? Langue française, 115(1), p. 75-87

Bonnet, L. (2017, 8 juin). Jean boyfriend : comment le porter ?. Femme actuelle, p.12

Charciarek, N. (2014, 26 septembre), Square Enix se lance dans le cloud gaming. Direct Matin p. 26.

Demoulin , A. ( 2014, 17 octobre). T'es pas Cape?. 20 Minutes, p. 20.

Deroy, L (1956). L'emprunt linguistique. Paris: Sociétés d'Editions, les Belles lettres.

Dubois, J (2002). Dictionnaire de linguistique. Paris: Larousse- Bordas.

En bref. (2014, 16 octobre). Direct Matin, p.26.

Enfin une vraie comédie des filles. (2014, 9 octobre). 20 Minutes, p.18.

Engstrøm, L (2005). Les Anglicismes dans le français Hexagonal. (Mémoire de Master inédite), Hosten, Université d'Oslo.

Fagard, B. (2010). Espace et grammaticalisation. L'évolution sémantique des prépositions dans les langues romanes. Editions Universitaires Européennes. p.442

Forlot, G (1999). Variation lexicale et reconstruction sémantique en migration : le cas de Français expatriés en milieu anglo-canadien. La linguistique, 35(2), 113-126.

Genolini, D. (2014, 10 octobre, 10). Saint du jour. Direct Matin, p.31.

Hanne, I, (2014, 9 octobre) Tentative de push. Libération, p.17

Haugen, E. (1950). The analysis of linguistic borrowing. Language, 26 (1), 210-231.

Humbley, J. (1974). Vers une typologie de l'emprunt linguistique. Cahiers de lexicologie, 25(1), 46-70.

Ils relookent leurs fans. (2014, 26 septembre). Direct Matin, p.22.

Japonnt, J. (2014, 26 septembre). Special running. Metro news, p.14

Jedlickova, K. (2009). Les emprunts dans la presse française contemporaine. (Thèse de doctorat), Brno, Université de Masaryk.

Joly, C. (2014, 2 octobre). Cinq raisons d'aller à Montreal. Metro news, p.12.

La censure selon Facebook (2014, 9 octobre). 20 Minutes, p.16.

La nouvelle ère des Bleus (2014, 16 octobre) Direct Matin, p.16.

La ville de Paris recrute des accompagnteurs. (2014, 29 septembre) 20 Minutes, p.11

Le bon plan du jour, (2014, 3 octobre). Direct Matin, p.31.

Le candidat Sarkozy expose ses idées. (2014, 3 Octobre). Direct Matin, p.14

Le mariage est à l'honneur ce week-end. 2014, 26 septembre). Direct Matin, p.22.

Le salon devient un terrain de jeu. (2014, 9 octobre). 20 Minutes, p.16.

Le vrai récit du week-end noir. (2014, 3 octobre). Direct Matin, p.10.

Les bons sports, (2014, 26 septembre). Metro news, p.1.

Loubier, Ch. (2011). De l'usage de l'emprunt linguistique. Montréal: Office québécois de la langue française.

Medi Sadoun. (2014, 21 septembre). Sa vie après le bon Dieu. Metro news, p.27.

On vous déroule le tapis rouge. (2014, 26 septembre). Direct Matin, p.24.

Ouadhi, S. (2014, 26 septembre). Je bosse comme si j'allais aux J.O. Metro news, p.21.

Paris en quête de Zénitude. (2014, 26 septembre). Direct Matin, p.18.

Paris XIXe. (2014, 21 septembre). Metro news, p.9.

Pas de Pub après 20h (2014, 3 octobre). Direct Matin, p.16.

Pédopornographie et terrorisme. (2014, 9 Octobre). 20 Minutes, p.16.

Picone, M. (1996). Anglicism, neologisms and dynamic French. Amesterdam: John Benjamins Publishing Company.

Prêts? Partez!. (2014, 26 septembre). Metro news, p.16.

Quartier des Batignolles. (2014, 10 octobre). Direct Matin, p.8.

Quefflec, A. (2000). , Emprunt ou xénisme : les apories d'une dichotomie introuvable ?", in Latin, D. et Poirier, C. (éds), Contacts de langues et. identités culturelles. Perspectives 
lexicographiques, Laval, Presses de l'Université Laval-Agence Universitaire de la Francophonie, pp. 283-300.

Rey-Debove, J. (1973). La sémiotique de l'emprunt lexical. Travaux de Linguistique et de Littérature, (11), 109-123.

Rey, A. (2008). Le français, une langue qui défie les siècles (Vol. 537). Gallimard.

Stars grâce au web. (2014, 10 octobre). Direct Matin, p.27.

Tournier, J. (1998). Les mots anglais du français. Paris: Belin.
Un super forfait mobile à prix mini. (2014, 9 octobre). 20 Minutes, p.5.

Un voyage culturel au cœur des cities, (2014, 26 septembre). Metro news, p.12.

Vinzerich, A. (2007). La sémantique du possible: approche linguistique, logique et traitement informatique dans les textes. (Thèse de doctorat inédite). Université de Paris IV - Sorbonne.

Walter, H. (2001). Honni soit qui mal y pense: l'incroyable histoire d'amour entre le français et l'anglais. Paris: Robert Laffont. 